\title{
Range Bias Modeling and Autocalibration of an UWB Positioning System
}

\author{
Andreas De Preter ${ }^{* \dagger}$, Glenn Goysens *, Jan Anthonis *, Jan Swevers ${ }^{\dagger \ddagger}$, Goele Pipeleers ${ }^{\dagger \ddagger}$ \\ * Octinion, Leuven-Heverlee, Belgium, \\ (e-mail: flastnamedoctinion.com) \\ $\dagger$ MECO Research Team, Dep. Mechanical Engineering, KU Leuven, Leuven, Belgium, \\ (e-mail: firstname.lastnameekuleuven.be) \\ $\ddagger$ DMMS lab, Flanders Make, Leuven, Belgium
}

\begin{abstract}
This paper describes two important challenges in the development of an ultra-wideband (UWB) positioning system. The first challenge is increasing the accuracy and robustness of the UWB technology. The range measurements are subject to disturbances, which introduce an unwanted bias. This range bias is measured, characterized and modeled to increase the positioning accuracy to an average of $3 \mathrm{~cm}$. The second challenge is a user-friendly deployment of the system. This is achieved by a semi-automated autocalibration procedure. In the proposed method the mobile device (tag) is moved around in the covered area. Based on all the captured data, the coordinates and a range bias model parameter of the static devices (anchors) are obtained. No additional measurement devices are required during the procedure. The positioning accuracy with the autocalibrated parameters decreases to an acceptable $9 \mathrm{~cm}$ on average, compared to the situation with exact parameters.
\end{abstract}

Index Terms-UWB, range bias modeling, sensor node calibration

\section{INTRODUCTION}

The problem of determining the location of an object in an open, outdoor environment can be achieved very robustly by using satellite based navigation systems. In such open spaces, these systems experience good signal reception from navigational satellites. However, satellite signals are strongly disturbed when they have to pass through leaves, trees, walls and other materials. Because of the weak signal quality, the satellite localization systems are unreliable and imprecise in non-open environments [1]. From this perspective, alternative technologies are being developed in order to solve the positioning problem in more challenging areas.

During the last decade, different technologies have been investigated to solve the localization problem within non-open environments. In city environments, the mobile communication network is often very dense. The position of a phone can be determined by fingerprinting or ranging, based on the signal power of the radio frequency (RF). The same applies for wireless local area networks (WLAN) in buildings [2]. A major advantage of these systems is that no additional infrastructure is required. The network coverage and availability determine highly the accuracy and reliability of these systems. Other

Andreas De Preter is supported by a Baekeland PhD scholarship (Grant 150712) through Flanders Innovation and Entrepreneurship (VLAIO).

978-1-7281-1788-1/19/\$31.00 @ 2019 IEEE technologies, e.g. RF identification (RFID) [3] and Bluetooth, are based on proximity sensing. Due to the limited ranging capacity, the accuracy and reliability of these technologies depend on the distribution of sensor nodes.

An RF-based network with sufficient ranging capacity is considered as an alternative to satellite-based systems for localization in non-open areas. In the selection of an appropriate RF-technology, the following requirements have to be taken into account: First, the signal needs to have a minimal penetration strength through objects. A clear line of sight between the network nodes and tracked object will never be guaranteed at every spot in a non-open area. Second, it must be possible to distinguish the main signal from disturbances caused by reflections and interferences. This ensures a robust measurement of the distance between network node and tracked node.

An interesting technology with respect to these requirements is ultra-wideband (UWB). The very wide bandwidth of this RF-signal offers interesting characteristics regarding the penetration through objects and main signal determination, as will be explained further in Sec. II-A. This technology is overall considered as a promising alternative for solving the positioning problem in environments with poor satellite reception. In contrast to these characteristics, there are still some important shortcomings that have to be overcome before this technology can be used as part of a reliable and viable positioning system. This paper tackles two of these shortcomings.

Despite the promising technology characteristics, it will be shown that the measured ranges suffer from a bias. Coping with this range bias is one of the most important challenges for robust and accurate positioning. The first contribution of this paper is a procedure to measure, characterize and model the range bias of an UWB system. This model adjusts the range measurements and increases the positioning accuracy.

The system must be easily deployable. A cumbersome calibration every time a new system is installed should be avoided. In this part, the challenge lies in a correct localization of the static network nodes. The second contribution of this paper is a semi-automated autocalibration procedure, which facilitates the deployment of the system.

The remainder of this paper is organized as follows. In Sec. II the state-of-the-art of the UWB technology and positioning 
algorithms is provided. Sec. III describes the range bias modeling and model based bias compensation for accurate positioning, and Sec. IV discusses the user-friendly autocalibration of the complete UWB setup.

\section{BASICS OF UWB POSITIONING}

\section{A. UWB technology}

From the start of the 21 st century, the ultra-wideband technology has gained a lot of interest in the field of indoor positioning systems [4]. The technology has been standardized in the IEEE 802.15.4 standard [5].

A UWB sensor transmits ultrashort pulses with a very low duty cycle, at a carrier frequency between $3.1 \mathrm{GHz}$ and 10.6 GHz. The short pulse duration allows to filter the signal more easily at the receiver side [6]. A consequence of the short pulse is that the signal has a very large bandwidth. It can therefore penetrate walls in buildings and can resolve individual multipath components [7].

Octinion $^{1}$ is developing an indoor positioning system for autonomous navigation of electric vehicles. It has developed its own navigation hardware (Fig. 1), which integrates the Decawave DW1000 chip, the world's first single-chip wireless transceiver based on ultra-wideband techniques [8]. The localization system will be used for autonomous navigation of agricultural robots.

\section{B. Radio Frequency Positioning}

When a signal is received, different techniques can be used to derive information necessary for positioning: the angle of arrival, received signal strength, and time of travel-based measures [9]. The estimation of the angle of arrival is not very interesting: it requires multiple antennas, which increases the receiver's complexity [10]. The received signal strength does not provide accurate data because of its strong dependency

${ }^{1}$ Octinion is an R\&D-company located in Leuven (Belgium) and specializes in automation in agriculture and food industry.

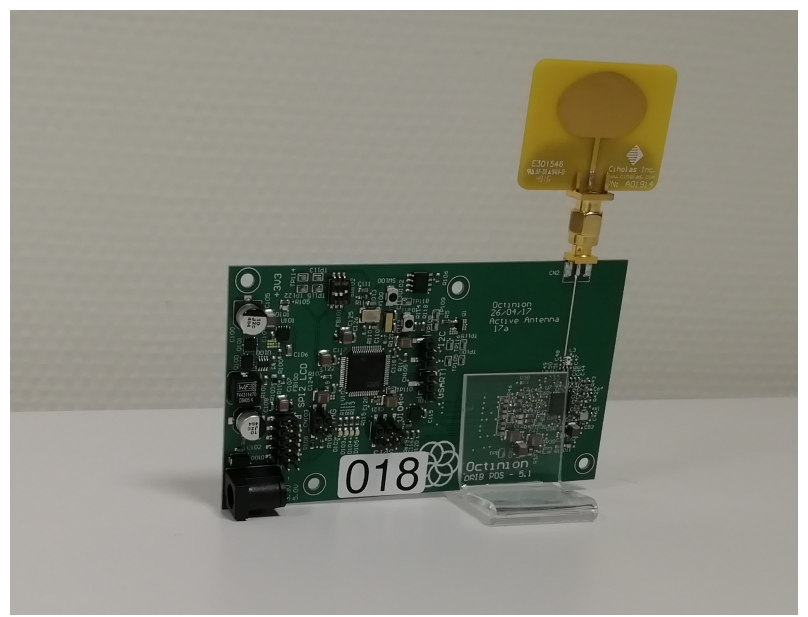

Fig. 1: Octinion navigation hardware with integrated Decawave DW1000 on the UWB channel parameters [10]. Due to the high time resolution of UWB signals, it is possible to determine the signal's transmission time very accurately, provided that the clocks of both transmitter and receiver are well-synchronized. The time of travel-based approaches are therefore the most interesting techniques in the context of localization with UWB [4].

The two most common time of travel-based approaches for localization are time of arrival (TOA) and time difference of arrival (TDOA) [9]. In order to determine an accurate distance from these measurements, the clocks of the hardware modules have to be synchronized. In the former situation, this is achieved by sending round-trip signals between two UWB nodes. Different ranging sequences have been investigated, whereof the double sided two-way ranging (DS-TWR) yields the most accurate result ([11], [12]). In the latter case, the clocks of the modules have to be synchronized with a direct link ([9], [10]).

The positioning technique discussed in this paper is the time of arrival method, with DS-TWR communication to achieve clock synchronization. This communication provides a range measurement between two modules which only need a power source. It is shown that the signal strength can be incorporated in the range bias model, in order to achieve more accurate results.

\section{Position estimation}

The mobile node (further called tag) communicates with the static network nodes (further called anchors). The measured distances between tag and anchors are combined by multilateration, a nonlinear least-squares problem, in order to obtain a position estimate. Many algorithms have been investigated to solve this optimization problem [13]. In case of an appropriate initial guess, the Newton-type methods have the fastest convergence [14].

In this paper the position is estimated through the Levenberg-Marquadt algorithm, whichs yields a stable and accurate convergence. As shown in Sec. III, the measured ranges are affected by a bias, which deteriorates the position estimate.

\section{RANGE BIAS MODELING}

\section{A. State of the art}

The localization performance of a positioning system is highly dependent on the quality of range measurements. For this reason, it is important to understand the diverse set of factors that can degrade ranging accuracy [15]. What follows in this subsection is a list of possible causes of range bias error.

A typical source of error is multipath. In this scenario the direct signal is influenced by the destructive and constructive interference of signals arriving at the receiver via different propagation paths [15]. The result of this interference can be quantified in a delay spread, which depends on the wave polarization and dielectric ground characteristics [16]. A range 


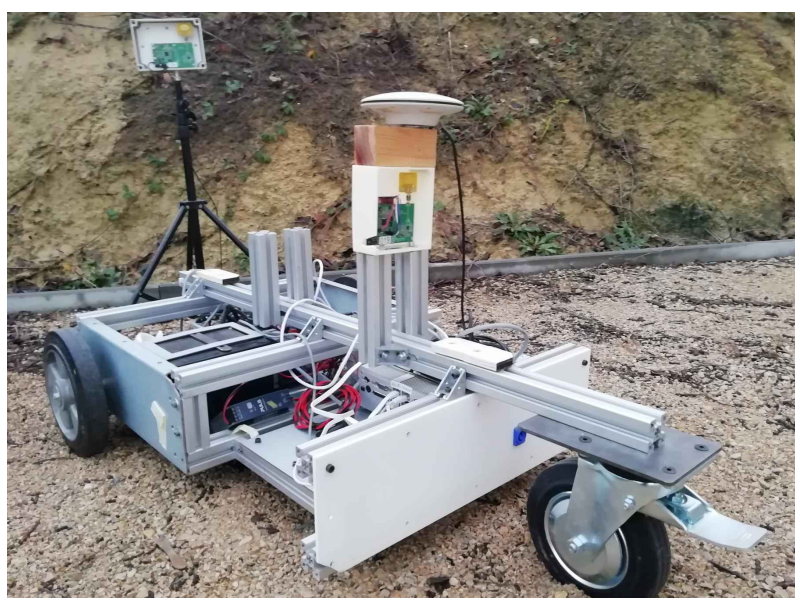

Fig. 2: Electric vehicle with UWB tag and RTK-GPS

bias model that attempts to capture this behavior was proposed in [17].

A similar problem appears in the situation where the direct path between sender and transmitter is blocked. Only reflected signals will arrive at the receiver, which will result in an overestimation of the range [18].

When a UWB signal is transmitted, a delay is introduced by the hardware [19]. This delay, which is different for each device, is caused by manufacturing differences, the surrounding circuitry, the antenna and even environmental effects (e.g. temperature). Despite the small value of the delay, it has significant impact on the range measurement. Although the delays of transmitter and receiver are different, they can be treated simultaneously as one, constant value to be estimated [20].

In other work, the range bias is more treated as a black box. The distance error is measured and an appropriate model has been fitted. In [21] the bias was modeled as a linear function with respect to the real distance.

This work will start with the same approach as in [21]. The range bias is measured accurately. Before looking for an appropriate model, some physical interpretation to the effects is provided. These insights will facilitate the search for a suitable model.

\section{B. Test description}

The range bias is investigated on its dependency with respect to the actual distance and the antenna orientation. The setup used for measuring the range bias is shown in Fig. 2. The robot is equipped with a UWB tag (see Sec. II-A) and an AsteRx-U RTK-GPS of Septentrio, which has a centimeterlevel accuracy. The bias on the range measurement is obtained by comparing the UWB ranges with 'exact' distances derived from the GPS coordinates. This methodology was also used in [22] and [23].

In order to generalize the results, the data of 2 anchors are used. The anchors are put on a line, whereon the test vehicle drives back and forth. After every test-run, the tag's antenna orientation is changed with $45^{\circ}$. The anchors' antennas are

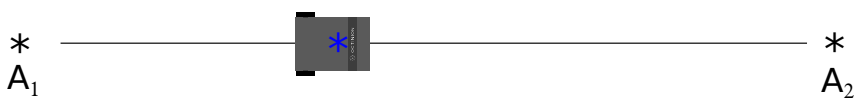

Fig. 3: Schematics of test decription

facing towards each other, in order to minimize their effect. A schematic of the experiment is shown in Fig. 3.

The results of the experiments are presented in Fig. 4 and Fig. 5. On the left side the range bias, calculated by comparing the RTK-GPS data with the UWB ranges, is shown as a function of the exact distance. On the right side an indicator of the received signal strength is plotted, also in function of the exact distance. This signal strength indicator is obtained by combining the first path amplitude values measured in the UWB chip. More information about this indicator can be found in [12].

\section{Measurement interpretation}

This subsection interprets the results of Fig. 4 and Fig. 5. The global trend in the range bias is similar for all experiments: the range bias increases until a certain distance has been reached. There it tends to converge to a constant value. As this phenomenon occurs equally in every test-run, it can be assumed that its origin lies within the UWB hardware and technology.

Apart from the global trend, some peculiar dips in the range bias are recorded. The effect occurs consistently in all experiments. Simultaneously a drop in signal strength is also measured. With this information, it can be derived that the dip in range bias is caused by multipath: the direct path signal suffers from destructive interference at the receiver by the signal reflected on the ground. Fig. 6 plots the theoretical Fresnel zones for the height conditions applicable during the experiments. It indicates the received power at the receiver when multipath with the ground surface occurs, for a signal frequency equal to the UWB carrier frequency. Although the large bandwidth of the UWB signal should decrease the multipath effect, it is still visible in the specified zones [16].

The orientation of the tag antenna has only an effect in the data of Fig. 4. Due to this inconsistency, it won't be taken into account in the remaining of this work.

Apart from the drops caused by the multipath effect, a decreasing trend is observed in the signal strength indicator. One can see that this is caused by the general path loss in wireless communication.

\section{Parameter estimation}

Before model parameters can be fitted on the measured data, a model structure has to be defined, which is unknown in advance. Two steps are followed in order to define this structure. 


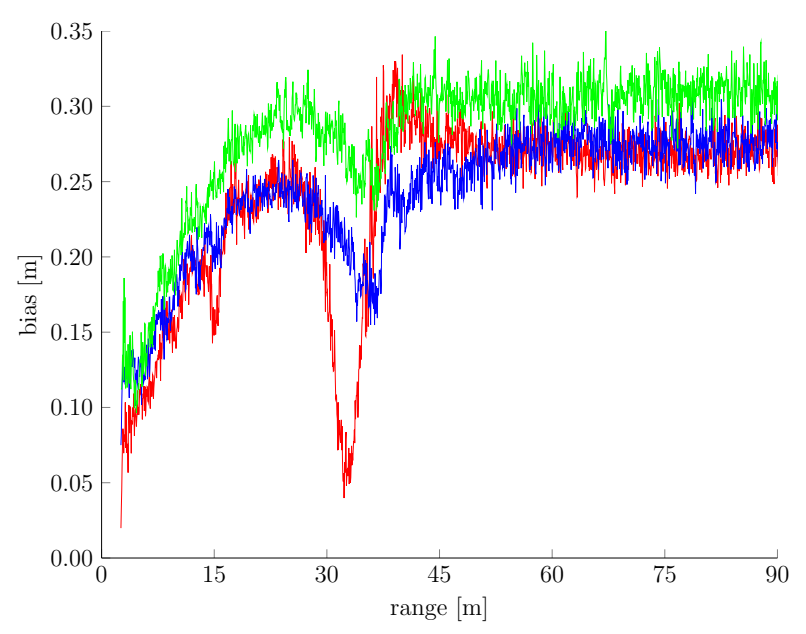

(a) range bias

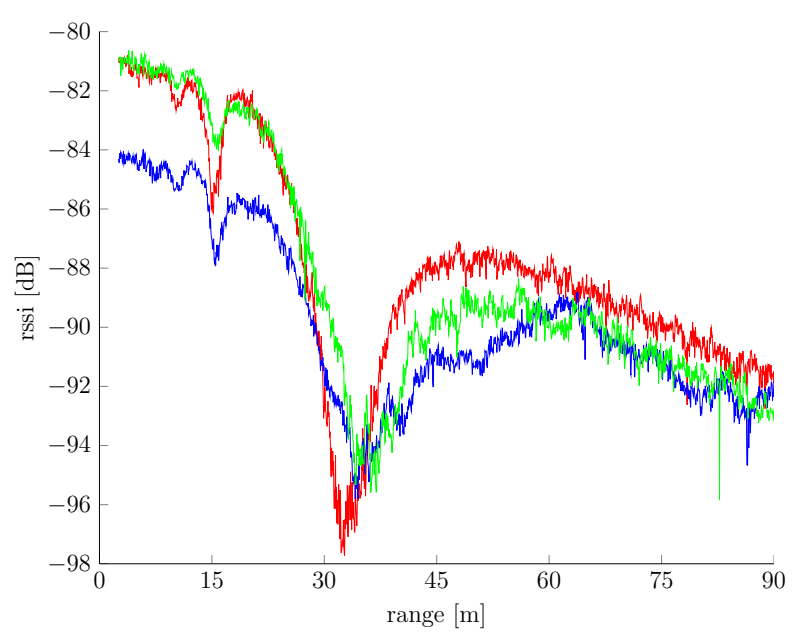

(b) signal strength

Fig. 4: Measured range bias and signal strength for $A_{1}$ in function of the tag's orientation $\left(-0^{\circ},-45^{\circ},-90^{\circ}\right)$

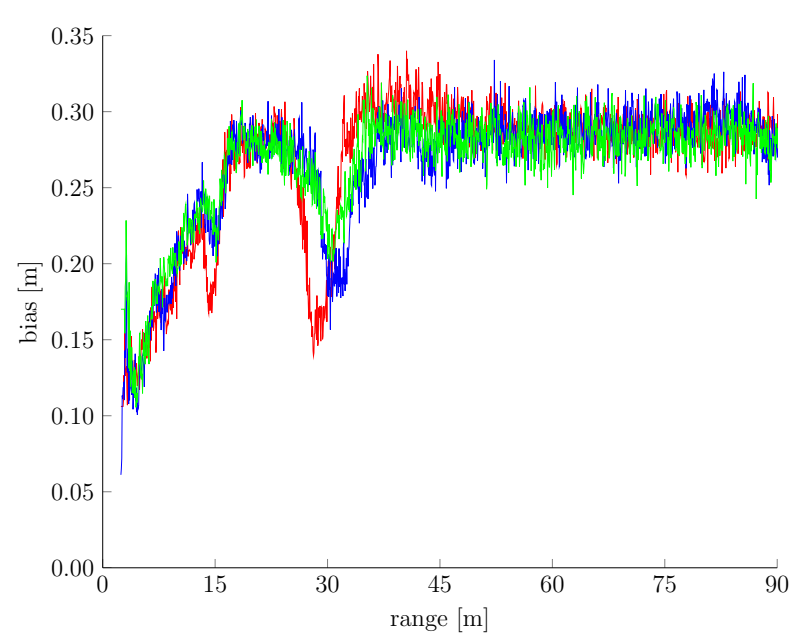

(a) range bias

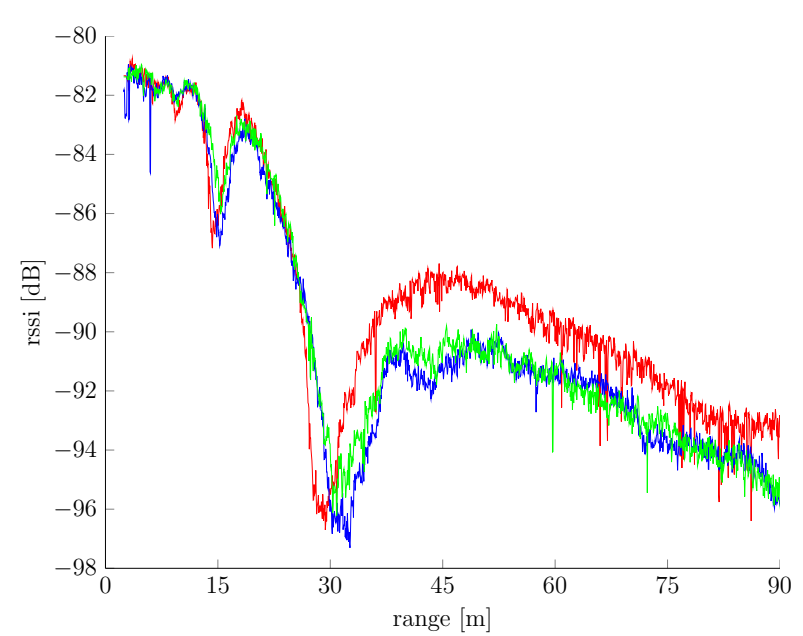

(b) signal strength

Fig. 5: Measured range bias and signal strength for $A_{2}$ in function of the tag's orientation $\left(-0^{\circ},-45^{\circ},-90^{\circ}\right)$

In the first step a purposely too complex model is selected. The parameters are estimated by an $l_{1}$-regularized least squares problem [24]. The optimization problem is defined as follows:

$$
\min _{p_{1}}\left\|\mathrm{RB}-f_{1}\left(p_{1}\right)\right\|_{2}^{2}+\lambda\left\|p_{1}\right\|_{1}
$$

with $\mathrm{RB}$ the measured range bias, $p_{1}$ the vector of unknown parameters, $f_{1}\left(p_{1}\right)$ the over-determined range bias model, and $\lambda \in \mathbb{R}^{+}$the regularization parameter. The latter acts as a design parameter: it regulates the trade-off between fitting the data and model complexity. The choice of $\lambda$ determines how complex the new model will be: only the most relevant parameters in the extensive model will remain non-zero. An overfit of the range bias is therefore avoided.

The optimal values $\hat{p}_{1}$, obtained by solving (1), are biased due to the penalization term: in order to have a better fit, they are shifted towards zero. In the second step all relevant parameters are re-estimated by minimizing the sum of squared residuals. The problem looks as follows:

$$
\min _{p_{2}}\left\|\mathrm{RB}-f_{2}\left(p_{2}\right)\right\|_{2}^{2}
$$

with $p_{2}$ and $f_{2}\left(p_{2}\right)$ the parameters and model structure which contains only the relevant terms determined by (1).

\section{E. Modeled range bias}

The previous subsection describes the methodology for obtaining an appropriate model of the range bias. This section shows the results of the parameter estimation.

The model for the range bias has the following structure for every anchor $j$ :

$$
\overline{\mathrm{RB}}_{j}=b_{j}+f(R, R S S i, p)
$$


with $b_{j}$ a constant value, and $f(R, R S S i, p)$ a function depending on the measured range $R$ and the indicator for received signal strength $R S S i$. The parameters $p$ used inside $f(R, R S S i, p)$ are equal for every anchor $j$. The parameter $b_{j}$ has to be determined for all anchors individually. As already mentioned in Sec. III-D, the tag orientation did not play a consistent role in the range bias. It is therefore not included in the general range bias model.

The modeled bias is in Fig. 7a compared with the exact bias, which was captured with the RTK-GPS. The remaining error is shown in Fig. 7b. The results are demonstrated with the data of anchor 2 with a tag orientation of $0^{\circ}$. The model depends on the measured range and the indicator for the received signal strength. The modeling of the range bias has an average error of $0.017 \mathrm{~m}$.

\section{F. Positioning results}

A correct modeling of the range bias increases the accuracy of the distance measurement between anchor and tag. The main goal of the UWB system is to combine all ranges into a position measurement of the tag. This section shows the impact of the range bias modeling on the positioning accuracy.

The position is calculated by multilateration of the distance measurements. The following problem is solved at every point:

$$
\min _{T}\left\|\left(R_{j}-\overline{\mathrm{RB}}_{j}\right)-d\left(A_{j}, T\right)\right\|_{2}^{2}
$$

with $R_{j}$ the measured range between anchor $A_{j}$ and tag $T$, $\overline{\mathrm{RB}}_{j}$ the modeled range bias, and $d\left(A_{j}, T\right)$ the Eucledian distance between $A_{j}$ and $T$. The coordinates of the tag $T$ are the unknown variables. The problem is solved by a LevenbergMarquadt iterative algorithm.

In order to validate the positioning results, a coverage trajectory was driven autonomously by the setup of Fig. 2. On an open area, 4 anchors were put in the corners of a rectangular field. The exact coordinates were measured by the RTK-GPS.

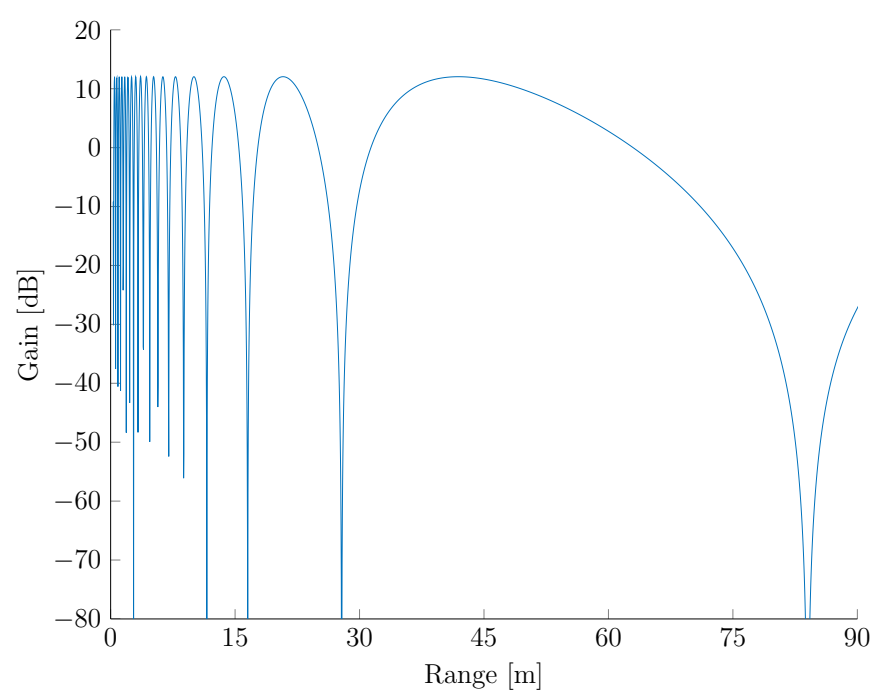

Fig. 6: Theoretical Fresnel zones for a signal on $3.9 \mathrm{GHz}$

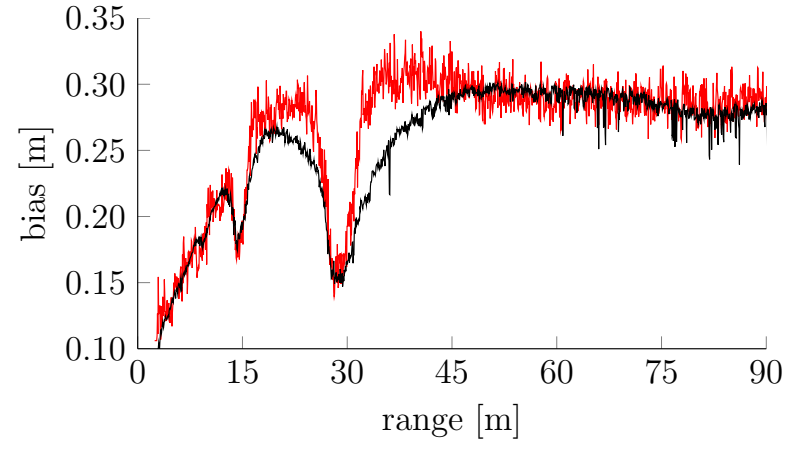

(a) measured range bias ( - ) and model $(-)$

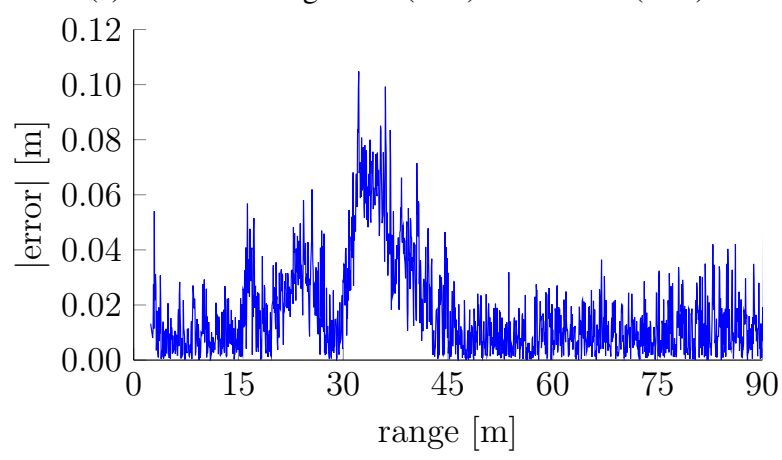

(b) modeling error

Fig. 7: Modeled range bias and remaining error for Anchor 2 with the $0^{\circ}$ tag orientation

For every anchor a calibration experiment, as described in Sec. III-B, was performed to identify the constant $b_{j}$ of the bias model.

The robot locates itself based on the UWB positioning system, while taking the identified range bias $\overline{\mathrm{RB}}_{j}$ for every anchor into account. This position measurement is used in the state-estimator, which combines wheel encoders and the UWB position in order to obtain the robot's state. The details of this implementation are out of scope of this work.

The resulting UWB coordinates estimation and the error with respect to the true location, measured with the RTK-GPS, are shown in Fig. 8. Afterwards, all the UWB range data was processed again for the situation where no range bias is taken into account. The average error in this case is $0.16 \mathrm{~m}$, which is improved significantly to $0.03 \mathrm{~m}$ when the range measurement is corrected for its bias. This second result is also shown in Fig. 8.

\section{ANCHOR AUTOCALIBRATION}

With the identification of an adequate range bias model in Sec. III, it is possible to localize a mobile robot with centimeter precision. The complete setup and calibration of the UWB positioning system is, however, very cumbersome: for every anchor, its coordinates have to be measured with RTK-GPS, and a calibration experiment (as described in Sec. III-B) has to be performed for estimating the constant bias between anchors 


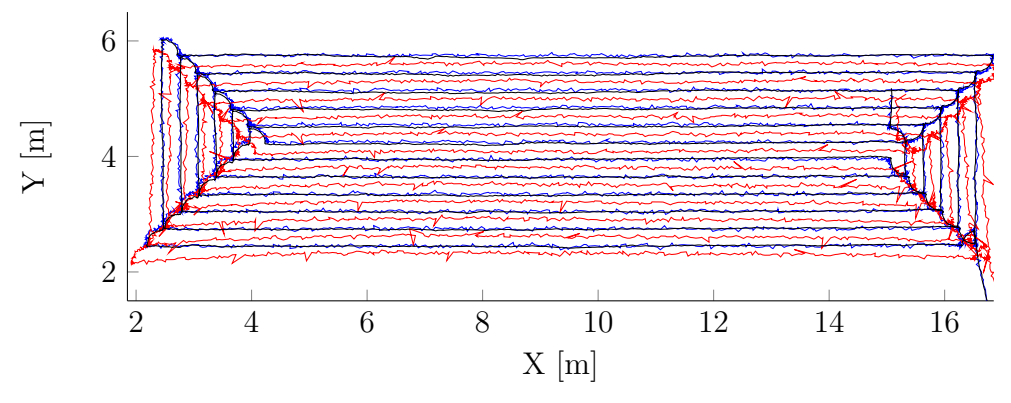

(a) Position

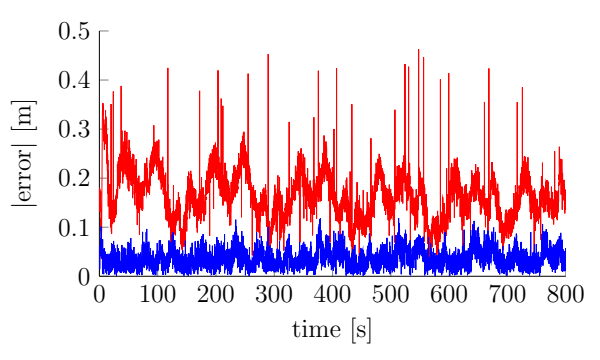

(b) Fault on position

Fig. 8: Position determination: — RTK-GPS, — no range bias correction, — with range bias correction

and tag. This section describes a method that identifies all these parameters without the need of an accurate GPS.

\section{A. State of the art}

The autocalibration of the anchors has already been covered frequently in literature. In [25] the node location is calculated with a maximum likelihood estimation (MLE) algorithm that uses time-of-arrival communication between the anchors and the tag, which is moved around in the area. Starting with an initial guess of the anchors and tag locations, the iterative algorithm converges to the actual position. During the last decade, research on anchor calibration focused on finding a good initial guess without any a priori information. If anchorto-anchor communication is possible, this could be solved with multidimensional scaling ([2], [26]). When only anchor-tag communication is available, the initial guess could be obtained with semidefinite programming [27], or, more recently developed, with algorithms based on minimal problems ([28]-[30]).

All recent work focuses on finding an initial guess for the MLE algorithm of [25]. The applicability of the MLE method is investigated in the following sections. The accurate determination of the anchor coordinates is performed with a dataset of ranges between the tag and anchors.

\section{B. Methodology}

This subsection describes the MLE algorithm for calibrating the anchors of the system described above. It is based on [25], where the method was applied on a positioning system with sound sensors.

The sensor network consists of $\mathrm{N}$ anchor nodes at unknown locations $A=\left\{A_{1}, A_{2}, \ldots, A_{N}\right\}$. The setup has one tag that is moved around in the area covered by the anchors. At $\mathrm{M}$ unknown locations the tag communicates with all the anchors which yields ranging data for each tag position $T=\left\{T_{1}, T_{2}, \ldots, T_{M}\right\}$. The ranging data with respect to every anchor consist of the actual distance and a bias, which has two parts: an unknown constant value, depending on the tag-anchor combination, and a part that depends on the range and received signal strength (see (3)). Because all the measurement data are captured with one tag, there are as many unknown biases as anchors: $b=\left\{b_{1}, b_{2}, \ldots, b_{N}\right\}$. The ranging measurement $R_{n, m}$ between anchor $A_{n}$ and tag $T_{m}$ is expressed as follows:

$$
R_{n, m}=d\left(A_{n}, T_{m}\right)+b_{n}+f\left(R_{n, m}, R S S i_{n, m}, p\right)+w
$$

with $w$ normally distributed noise $\mathcal{N}\left(0, \sigma_{R}^{2}\right), d\left(A_{n}, T_{m}\right)$ the Euclidean distance between $A_{n}$ and $T_{m}$, and $b_{n}+$ $f\left(R_{n, m}, R S S i_{n, m}, p\right)$ the modeled range bias from Sec. III-E. The formulation is valid for both $2 \mathrm{D}$ and $3 \mathrm{D}$ calibration, which will include 2 or 3 unknown coordinates per tag and anchor location respectively. However, when the tag and anchors height are similar, the 3D-calibration problem becomes very ill-conditioned.

In order to calculate simultaneously the constant biases $b$, the anchor coordinates $A$ and the tag coordinates $T$, the following objective has to be minimized:

$$
\left\|\left(R_{m, n}-b_{n}-f\left(R_{m, n}, R S S i_{m, n}, p\right)\right)-d\left(A_{n}, T_{m}\right)\right\|_{2}^{2}
$$

while taking every combination of $n=1, \ldots, N$ and $m=$ $1, \ldots, M$, with every $b_{n}, A_{n}$ and $T_{m}$ as free variables.

The objective is a non-linear least squares problem formulation which can be minimized with Gauss-Newton. It is highly non-convex and could get stuck easily in local minima if an initial guess is not chosen well [27].

The initial guess can be obtained as follows: by switching the anchors to an anchor-to-anchor communication mode, the intermediate distances can be obtained. By solving the multilateration equations, the anchor coordinates can be calculated. In order to fix the anchor coordinate system, its origin is put in $A_{1}$, while the x-axis goes through a different, arbitrarily chosen anchor [25]. The intermediate anchor distances also suffer from the bias phenomenon discussed in Sec. III. However, the results are only used as initial guess for the autocalibration problem. If the latter is well-conditioned, the coordinates should converge to their exact values.

\section{Autocalibration results}

The mobile robot drives around in an open, flat area. The anchors' height is measured with tape measure. Therefore the autocalibration problem is limited to the $2 \mathrm{D}$ case.

In the next situation there are four anchors located at the corners of an area, as already explained in Sec. III-F. The 
TABLE I: Calibration results

\begin{tabular}{|c||c|}
\hline Parameter & Error $[\mathrm{m}]$ \\
\hline \hline$b_{1}$ & 0.09 \\
\hline \hline$A_{2 x}$ & 0.15 \\
$A_{2 y}$ & 0.08 \\
$b_{2}$ & 0.0 \\
\hline \hline$A_{3 x}$ & 0.18 \\
$A_{3 y}$ & 0.02 \\
$b_{3}$ & 0.03 \\
\hline \hline$A_{4 x}$ & 0.05 \\
$b_{4}$ & 0.02 \\
\hline
\end{tabular}

positioning system has initially no information on the anchorstag constant range biases nor the anchors' coordinates. The tag travels a path in the covered area. Its trajectory is calculated by multilateration, which is based on the initial estimation of the anchor locations and assumes zero biases [13]. The initial estimation of the anchor locations, the zero biases, and the results of the multilateration are used as "initial guess" for the MLE calibration method.

The dataset used for autocalibration is obtained by driving a widespread pattern in the area of interest. This guarantees the best convergence for estimating the anchors' coordinates [31]. The trajectory is plotted in Fig. 9.

The fault on the parameter estimation, resulting from the autocalibration procedure, are shown in Table I. Despite the many degrees of freedom, the parameters converge quite well to their exact value.

\section{Positioning with autocalibrated parameters}

Although there are differences between the exact and estimated parameters (see Table I), it is the actual positioning by combining all ranges that matters when quantifying the system's performance. In order to have an idea of the impact of these differences, all positions in the experiment from

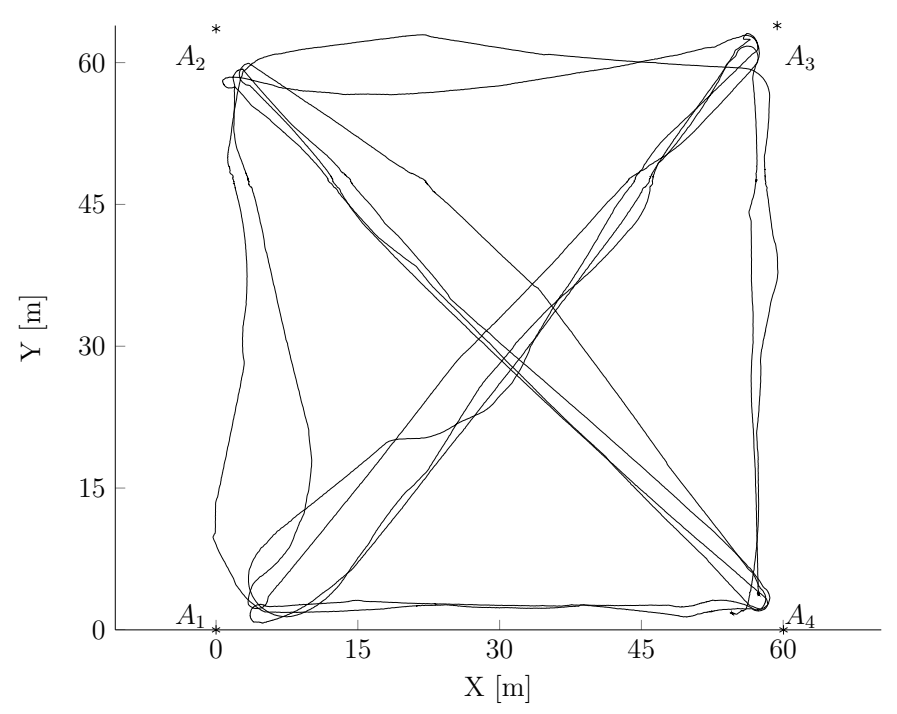

Fig. 9: Trajectory of autocalibration experiment
Sec. III-F are recalculated with the estimated parameters. The results are shown in Fig. 10.

The average positioning error when using the estimated parameters is $0.09 \mathrm{~m}$, which is still significantly larger than the situation with exact parameters $(0.03 \mathrm{~m})$. This could be caused by the fact that, despite all the effort, the range bias is not yet perfectly modeled (see Fig. 7). As shown in [31], the parameters estimated by (6) should converge to their exact value, when only Gaussian noise is present on the modeled range.

\section{CONCLUSION}

This paper discusses two challenges in the development of an UWB positioning system. The first challenge is improving accuracy through accurate bias modeling and identification. The range bias is measured accurately with an RTK-GPS, and characterized by an indicator value for the signal strength. Further investigation is required to incorporate the (subtle) effect caused by antenna orientation. With an $l_{1}$-regularized least squares optimization problem, the relevant model parameters are identified. These parameters are finally estimated with a regular least-squares problem. The resulting positions are compared with the situation where no range bias was taken into account.

The second challenge is to setup and calibrate the system in a user-friendly way. A method is proposed which only requires a rough estimate of the anchors' coordinates. The tag is moved around in the area covered by the anchors. With an optimization problem, all anchor coordinates and the constant bias between the anchors and tag are estimated simultaneously. Due to the remaining deficit in the range bias modeling, the parameters don't converge to their exact value. However, positioning with the estimated values has still an acceptable accuracy.

\section{REFERENCES}

[1] M. Andreotti, M. Aquino, M. Woolfson, J. Walker, and T. Moore, "Signal propagation analysis and signature extraction for gnss indoor positioning," in Record - IEEE PLANS, Position Location and Navigation Symposium, vol. 2006, 2006, pp. 913-919.

[2] Z. Yonghao, W.-C. Wong, and H. Garg, "A calibration-free indoor localization system using pseudo-distances in wlan environments," in Proc. IEEE Indoor Positioning and Indoor Navigation Conference (IPIN 2017), Sep. 2017, pp. 1-8.

[3] V. Gharat, E. Colin, G. Baudoin, and D. Richard, "Indoor performance analysis of lf-rfid based positioning system: Comparison with uhf-rfid and uwb," in Proc. IEEE Indoor Positioning and Indoor Navigation Conference (IPIN 2017), Sep. 2017, pp. 1-8.

[4] S. Gezici, T. Zhi, G. Giannakis, H. Kobayashi, A. Molisch, H. Poor, and Z. Sahinoglu, "Localization via ultra-wideband radios: a look at positioning aspects for future sensor networks," IEEE Signal Processing Magazine, vol. 22, no. 4, pp. 70-84, Jul. 2005.

[5] IEEE Standard for Low-Rate Wireless Networks (802.15.4-2015). USA: IEEE, 2016

[6] H. Liu, H. Darabi, P. Banarjee, and J. Liu, "Survey of wireless indoor positioning techniques and systems," IEEE Transactions on Systems, Man, and Cybernetics, Part C (Applications and Reviews), vol. 37, no. 6, pp. 1067-1080, Nov. 2007.

[7] J. Ruiz, A. Ramon, and F. Granja, "Comparing ubisense, bespoon, and decawave uwb location systems: Indoor performance analysis," IEEE Transactions on Instrumentation and Measurement, vol. 66, no. 8, pp. 2016-2117, Aug. 2017. 


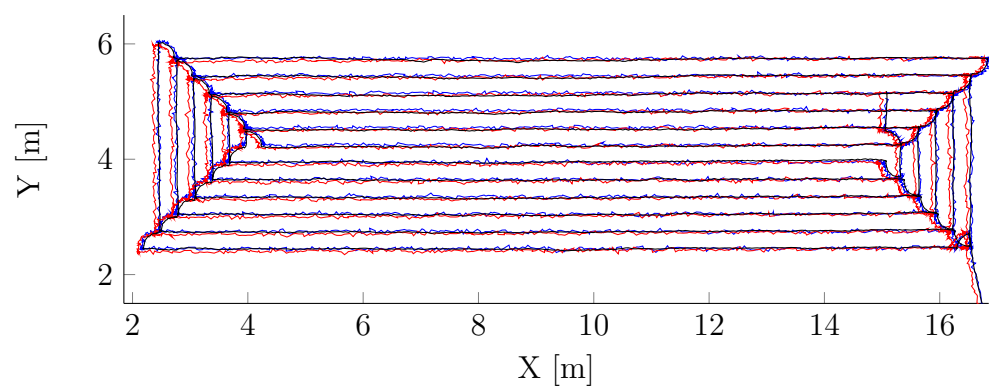

(a) Position

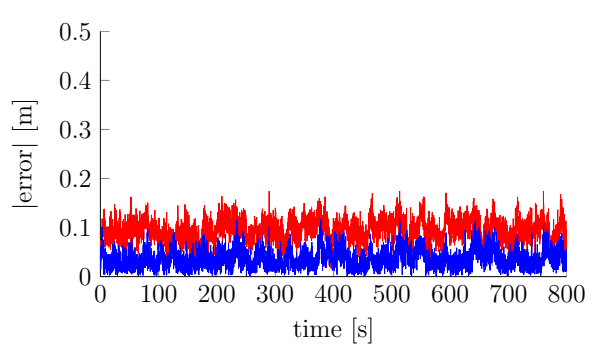

(b) Fault on position

Fig. 10: Position determination: — RTK-GPS, —estimated parameters, —exact parameters

[8] "Dw1000 radio ic," https://www.decawave.com/product/dw1000-radioic/, accessed: 2019-05-01.

[9] S. Goswami, Indoor Location Technologies. Springer New York, 2013

[10] H. Soganci, S. Gezici, and H. V. Poor, "Accurate positioning in ultrawideband systems," IEEE Wireless Communications, vol. 18, no. 2, pp. 19-27, 2011.

[11] M. Kwak and J. Chong, "A new two-way ranging algorithm for ranging system," in Proc. IEEE International Conference on Network Infrastructure and Digital Content (IC-NIDC2010), Sep. 2010, pp. 470473.

[12] DW1000 User Manual, Decawave Ltd., 2015, version 2.06.

[13] J. Yan, "Algorithms for indoor positioning systems using ultra-wideband signals," Ph.D. dissertation, Delft University of Technology, Netherlands, Dec. 2010. [Online]. Available: https://repository.tudelft.nl/islandora/object/uuid:013544ae-61574479-bb1e-be81f63461f2

[14] J. Wendeberg, F. Hflinger, C. Schindelhauer, and L. Reindl, "Calibrationfree tdoa self-localisation," Journal of Location Based Services, vol. 7, no. 2, pp. 121-144, May 2013.

[15] D. Dardari, A. Conti, U. Ferner, A. Giorgetti, and M. Win, "Ranging with ultrawide bandwidth signals in multipath environments," Proceedings of the IEEE, vol. 97, no. 2, pp. 404-426, 2009.

[16] S. Tanchotikul, P. Supannakoon, S. Promwong, and J. Takada, "Rms delay spread estimation of ground reflection channel for uwb communications," in IEEE International Symposium on Communications and Information Technology, 2005. ISCIT 2005, vol. 2. IEEE, 2005, pp. 1117-1120.

[17] B. Alavi, K. Pahlavan, N. Alsindi, and X. Li, "Indoor geolocation distance error modeling using uwb channel measurements," in IEEE International Symposium on Personal, Indoor and Mobile Radio Communications, PIMRC, vol. 1, 2005, pp. 481-485.

[18] İ. Güvenç, C.-C. Chong, F. Watanabe, and H. Inamura, "Nlos identification and weighted least-squares localization for uwb systems using multipath channel statistics," EURASIP Journal on Advances in Signal Processing, vol. 2008, no. 1, pp. 1-14, 2007.

[19] APSO14 Application Note - Antenna Delay Calibration of DW1000based Products and Systems, Decawave Ltd., 2014, version 1.01.

[20] K. Horváth, G. Ill, and Á. Milánkovich, "Calibration method of antenna delays for uwb-based localization systems," in Proc. IEEE International
Conference on Ubiquitous Wireless Broadband (ICUWB 2010), Sep. 2010.

[21] S. Monica and G. Ferrari, "Improving uwb-based localization in iot scenarios with statistical models of distance error." Sensors (Basel, Switzerland), vol. 18, no. 5, 2018. [Online]. Available: http://search.proquest.com/docview/2041625646/

[22] Z. Koppanyi, C. Toth, D. Grejner-Brzezinska, and G. Jkw, "Performance analysis of uwb technology for indoor positioning," in Institute of Navigation International Technical Meeting 2014, ITM 2014, 2014.

[23] K. Dierenbach, S. Ostrowski, G. Jkw, C. Toth, D. Grejner-Brzezinska and Z. Koppanyi, "Uwb for navigation in gnss compromised environments," 2015.

[24] H. Ohlsson, "Regularization for sparseness and smoothness: Applications in system identification and signal processing," $\mathrm{Ph} . \mathrm{D}$. dissertation, Linköpings Universitet, 2010.

[25] R. Biswas and S. Thrun, "A passive approach to sensor network localization," in Proc. IEEE International Conference on Intelligent Robots and Systems (IROS 2004), vol. 2, Sendai, Japan, Sep. 2004, pp. 1544-1549.

[26] Y. Shang, W. Ruml, Y. Zhang, and M. Fromherz, "Localization from mere connectivity," in Proc. 4th ACM international symposium on mobile ad hoc networking \& computing, Jun. 2013, pp. 201-212.

[27] P. Biswas, T.-C. Lian, T.-C. Wang, and Y. Ye, "Semidefinite programming based algorithms for sensor network localization," in Proc. ACM Transactions on Sensor Networks, vol. 2, no. 2, May 2006, pp. 188-220.

[28] K. Batstone, M. Oskarsson, and K. Åström, "Robust time-of-arrival self calibration with missing data and outliers," in Proc. 24th European Signal Processing Conference (EUSIPCO 2016), 2016, pp. 2370-2374.

[29] — "Robust time-of-arrival self calibration and indoor localization using wi-fi round-trip time measurements," in Proc. IEEE Workshop on Advances in Network Localization and Navigation (ANLN 2016), May 2016, pp. 3875-3879.

[30] — , "Towards real-time time-of-arrival self-calibration using ultrawideband anchors," in Proc. IEEE Indoor Positioning and Indoor Navigation Conference (IPIN 2017), Sep. 2017, pp. 1-8.

[31] A. De Preter, J. Anthonis, J. Swevers, and G. Pipeleers, "Experiment design for ultra-wideband sensor node calibration," in WIP Indoor Positioning and Indoor Navigation Conference (IPIN 2018), 2018. [Online]. Available: http://ipin2018.ifsttar.fr/program/poster-listmenu/\#c20352 